\title{
The Optimal Analysis of Default Probability for a Credit Risk Model
}

\author{
Aiyin Wang, ${ }^{1,2}$ Ls Yong, ${ }^{1}$ Weili Zeng, ${ }^{1}$ and Yang Wang ${ }^{1}$ \\ ${ }^{1}$ The School of Finance, Southwestern University of Finance and Economics, Chengdu 610074, China \\ ${ }^{2}$ Department of Mathematics, Xinjiang University of Finance and Economics, Urumqi 830012, China
}

Correspondence should be addressed to Aiyin Wang; way8848@sina.com

Received 2 January 2014; Accepted 9 February 2014; Published 17 March 2014

Academic Editor: Sheng-Jie Li

Copyright (C) 2014 Aiyin Wang et al. This is an open access article distributed under the Creative Commons Attribution License, which permits unrestricted use, distribution, and reproduction in any medium, provided the original work is properly cited.

A credit risk mathematical model is investigated. Under regular conditions, a different recovery scheme is proposed, which is an extension of the recovery of treasury value scheme (RTV) with time-continuous liquidation. Assuming that a function depends on the optimal time for the liquidation and the recovery rate, we obtain the functional expression of the risky bond price. When the firm value follows a jump-diffusion process with a Log-exponentially distributed jump, we develop a method to obtain the optimal default probability with time-continuous liquidation.

\section{Introduction}

The structural model and the reduced form model are two basic models for valuing credit risk of corporate bonds. The structural model, pioneered by Merton [1,2], illustrates the firm-value process and the default threshold. By assuming that the evolution of firm value follows a diffusion process, Merton [2] obtained the mean recovery rate, the probability of default and the implied credit spread of a corporate bond. Black and Cox [3], Lonigstaff and Schwartz [4], and Leland [5] modified and extended Merton's model in many ways except the diffusion process. Jones et al. [6], Sarig and Warga [7], and Fons [8] found that as a sudden drop in firm value is excluded, their credit spread is smaller than the observed market spread. Zhou [9] proposes a jump-diffusion firmvalue process and obtains a much closer credit spread. The reduced-form model, adopted by Artzner and Delbaen [10], Jarrow and Turnbull [11], Jarrow et al. [12], Li [13], and Madan and Unal [14] does not treat the relation between firm value and default in an explicit way. It is in an unpredictable way. The observed market credit spread is used in the reducedform model to obtain the optimal probability of default and the mean recovery rate.

Chen and Panjer [15] unify the structural model and the reduced-form model by showing the equivalence of yield spreads, which leads to the use of ruin theory in credit risk. Ruin theory was firstly applied to credit rating by Yang [16] who used a pure jump process. Different from those in Yang [16], Chen and Panjer [17] assumed that the credit spread is in accord with the observed market credit risk. Chen and Panjer [17] assumed that the credit spread is in accord with the observed market credit spread, which is used to obtain the implied jump distribution. Chen and Panjer [17] make another specific assumption that the firm can only be liquidated at the maturity of the bond, once default occurs before it, which is far more different from the reality. Motivated by the desire to extend the work of Chen and Panjer [17], we relax its assumption and study a credit risk valuation model with time-continuous liquidation.

In this paper, a recovery scheme, which is an extension of the recovery of treasury value scheme (RTV) with timecontinuous liquidation, is used under regular conditions. By using the relationship of the required time for liquidation and the recovery rate, we obtain the function expression of the risky bond price. In addition, a relationship between credit risk and ruin theory is constructed by using equivalence principle. Thus, the methodology in ruin theory can be used in credit valuation. Furthermore, when the firm value 
follows a jump-diffusion process with a Log-exponentially distributed jump, we develop a method to obtain the optimal default probability with time-continuous liquidation.

This paper is organized as follows. Section 2 introduces a new recovery scheme by introducing time-continuous liquidation. Section 3 introduces the structural model when the firm value follows a jump-diffusion process. In Section 4, the relationship between the ruin theory and credit risk is established by using equivalence principle. In Section 5, we obtain the credit spread by assuming that the firm value follows a jump-diffusion process with Log-exponentially distributed jumps. Two seniorities cases are considered in Section 6. In Section 7, we propose a method to find the implied jump size distribution and default probability caused by a jump. Finally, we draw some conclusions.

\section{The Recovery Scheme}

There are three major recovery schemes in credit risk literature, which are recovery of par value (RPV), recovery of treasury value (RTV), and recovery of market value (RMV). Here we use a different recovery scheme by introducing timecontinuous liquidation.

Let $P(t, T)$ denote the time-t price of a risk-free zerocoupon bond paying one dollar at maturity time $T$, where $0 \leq t \leq T<\infty . V(t, T)$ denotes the time- $t$ price of a risk corporate bond paying one dollar at maturity time $T$. Let $r(t, T)$ and $R(t, T)$ represent the yield to maturity on the riskfree bond and risky bond, respectively. The bond prices are written as

$$
\begin{gathered}
P(t, T)=e^{-r(t, T) *(T-t)}, \\
V(t, T)=e^{-R(t, T) *(T-t)} .
\end{gathered}
$$

We make the following assumptions.

Assumption I. We have the following.

(1) There exists a risk-neutral measure $Q$ so that arbitrage opportunities are excluded.

(2) There is no liquidation cost.

(3) The recovery rate, which is denoted by $\omega(\tau)$, is the ratio of the market value of the firm at the time of default $\tau$ to its debt obligation.

(4) The value of the firm remains steady after default so that recovery rate at the time of default $\omega(\tau)$ is equal to the recovery rate at the time of liquidation $\omega(\widehat{\tau})$.

(5) The short interest rate is flat and fixed and denoted by $r$.

(6) The defaulted firm can be liquidated at any time before maturity $T$. The time of liquidation is denoted by $\widehat{\tau}$.

The first five assumptions can be found in [17] and assumption 6 is our own.

In no-arbitrage theory, a risky bond of one-dollar face amount can be regarded as a contingent claim paying the recovery rate if default dose happens and paying one dollar if it does not. Under the risk-neutral measure $Q$, the time- $t$ price of risky bond is the expected value of contingent claim at time- $t$.

From the assumption, we propose a recovery scheme, named $\mathrm{RTV}^{+}$as follows. When a default event happens and the firm can be liquidated at any time before the time of maturity of the bond, the bond holders get a fractional payment of face amount. The remaining value of the defaulted firm does not earn any interest during the period from default time to liquidation. Under $\mathrm{RTV}^{+}$scheme, the recovery rate at the time of the liquidation $\widehat{\tau}$ is equal to a payment at the time of default $\tau$ of an amount namely, the recovery rate times the treasure bond price $P(\widehat{\tau}, T)$. Under risk-neutral valuation a risky bond price for a $\mathrm{RTV}^{+}$scheme can be written as

$$
\begin{array}{r}
V(t, T)=E_{t}^{\mathrm{Q}}\left[P(t, \widehat{\tau}) \cdot \omega(\tau) \cdot I_{(\tau \leq T \mid \tau>T)}\right. \\
\left.+P(t, T) \cdot I_{(\tau>T \mid \tau>t)}\right],
\end{array}
$$

where $E_{t}^{Q}$ denotes the conditional expectation at time $t$ under the risk neutral measure $Q$ and $I$ is an conditional indicator function which is

$$
\begin{aligned}
& I_{(\tau \leq T \mid \tau>t)}= \begin{cases}1, & \tau \leq T, \\
0, & \text { otherwise, }\end{cases} \\
& I_{(\tau \geq T \mid \tau>t)}= \begin{cases}1, & \tau>T, \\
0, & \text { otherwise. }\end{cases}
\end{aligned}
$$

In no-arbitrage theory, for a risk-free bond, we have

$$
P(t, \widehat{\tau})=P(t, T) \cdot e^{-r(T-\widehat{\tau})} .
$$

Assumption II. Let required time period for liquidation ( $\widehat{\tau}-$ $\tau$ ) be proportional to the recovery rate $\omega(\tau)$. It means that the more the value of defaulted firm remains, the more the time is required for liquidation.

As a result, the time period $(T-\widehat{\tau})$ is inversely proportional to the recovery rate $\omega(\tau)$. To facilitate the calculation, we assume

$$
T-\widehat{\tau}=-\frac{1}{r} \ln [\omega(\tau)]
$$

Substituting (4) and (5) into (2) yields

$$
\begin{array}{r}
V(t, T)=E_{t}^{Q}\left[P(t, T) \cdot \omega^{2}(\tau) \cdot I_{(\tau \leq T \mid \tau>T)}\right. \\
\left.+P(t, T) \cdot I_{(\tau>T \mid \tau>t)}\right] .
\end{array}
$$

If the risk-free rate $r$ is independent of the default process, we have

$$
V(t, T)=P(t, T) \cdot E_{t}^{Q}[\omega],
$$

where

$$
\omega= \begin{cases}1, & \tau>T, \\ \omega^{2}(\tau), & t<\tau \leq T .\end{cases}
$$


Taking conditional expectation on the random indication $I_{(\tau \leq T \mid \tau>T)}$, we have

$$
\begin{gathered}
V(t, T)=P(t, T) \cdot E_{t}^{Q}\left\{E_{t}^{Q}\left[\omega \mid I_{(\tau \leq T \mid \tau>t)}\right]\right\} \\
=P(t, T) \cdot\left\{E_{t}^{Q}\left[\omega \mid I_{(\tau \leq T \mid \tau>t)}=0\right]\right. \\
\cdot Q\left(I_{(\tau \leq T \mid \tau>t)}=0\right) \\
+E_{t}^{Q}\left[\omega \mid I_{(\tau \leq T \mid \tau>t)}=1\right] \\
\left.\cdot Q\left(I_{(\tau \leq T \mid \tau>t)}=1\right)\right\} \\
=P(t, T) \cdot\{1 \cdot Q(\tau>T \mid \tau>t) \\
\left.+E_{t}^{Q}\left[\omega^{2}(\tau)\right] \cdot Q(\tau \leq T \mid \tau>t)\right\} \\
=P(t, T) \cdot\left\{1-Q(\tau \leq T \mid \tau>t)+E_{t}^{Q}\left[\omega^{2}(\tau)\right]\right. \\
\cdot Q(\tau \leq T \mid \tau>t)\} \\
=P(t, T) \cdot\left\{1-\left\{1-E_{t}^{Q}\left[\omega^{2}(\tau)\right]\right\}\right. \\
\cdot Q(\tau \leq T \mid \tau>t)\} .
\end{gathered}
$$

Taking the log operation for the both sides of (9), we obtain the yield spread:

$$
\begin{array}{r}
R(t, T)-r(t, T)=-\frac{1}{T-t} \ln \left\{1-\left\{1-E_{t}^{\mathrm{Q}}\left[\omega^{2}(\tau)\right]\right\}\right. \\
\cdot Q(\tau \leq T \mid \tau>t)\} .
\end{array}
$$

For both structural model and reduced-form model, we know that (10) holds. We assume that the corresponding credit spread is consistent with the market credit spread. Thus, we can use the market spread to obtain the default probability and the recovery rate.

\section{The Structural Model with a Jump-Diffusion Firm-Value Process}

Let us state the following assumption which can be found in Chen and Panjer [17].

Assumption III. We have the following.

(1) The market is perfect and frictionless with no transaction cost, which means that there are many sellers as well as buyers who can trade as much as they want in continuous time.

(2) The short interest rate is flat and fixed.

(3) The Modigliani-Miller theorem holds so that the firm value is invariant to its capital structure.

(4) The firm value follows the jump-diffusion process proposed by Merton [2]. Let the capital asset pricing model (CAPM) hold for equilibrium returns. Thus the expected instantaneous return of the firm value equals risk-free rate $r$. The diffusion process represents systematic risk and the jump components represent nonsystematic risk which has zero beta.

(5) The firm issues both equities and bonds. The bonds mature at the same time but differ only in the priority with which they are redeemed.

Let $V$ represent the total market value of the firm. The firm value $V_{t}$ at time- $t$ is equivalent to the sum of the time$t$ market price of the equity and debt obligations. Under Assumption III, the dynamics of the firm value are defined by the following jump-diffusion process:

$$
\frac{d V_{t}}{V_{t}}=\left(r-\lambda_{\mu_{J}}\right) d t+\sigma d Z_{t}+J_{N_{t}} d N_{t}
$$

where $V_{t}$ is the firm value at time $t, \sigma^{2}$ is instantaneous variance, $r$ is instantaneous return, $Z_{t}$ is a standard Brownian motion, $N_{t}$ is total number of jumps up to time- $t$, and $J_{N_{t}}$ is the jump size as a proportion of $V_{t}$ of $N_{t}$ th jump. $N_{t}$ follows a Poisson process with parameter $\lambda$ and is stochastically independent of $Z_{t} . J_{N_{t}}$ is assumed to be independent of $\left(N_{t}\right)_{t \geq 0}$ and is independently and identically distributed whose moment generating function exists with mean $\mu_{j}$ and variance $\sigma_{j}^{2}$. The mean value of the instantaneous change of the firm value is $E\left[d V_{t}\right]=r V_{t} d t$. The percentage change of the firm value $d V_{t} / V_{t}$ as well as the jump amplitude $J_{i}$ is bounded in $[-1,+\infty]$. If we assume that $Y$ is a nonnegative random variable and $Y_{i}=J_{i}+1$, for $i=1$ to $N_{t}$, then $Y_{i}$ falls in $[0,+\infty]$. Let $Y(t)=1$ when $N_{t}=0$, and

$$
Y(t)=\prod_{i=1}^{N_{t}} Y_{i}=\prod_{i=1}^{N_{t}}\left(J_{i}+1\right),
$$

where $N_{t} \neq 0$. From Itô's lemma and Zhou [9], we have

$$
d \ln V_{t}=\left(r-\frac{\sigma^{2}}{2}-\lambda_{\mu_{J}}\right) d t+\sigma d Z_{t}+\ln Y_{N_{t}} d N_{t} .
$$

\section{The Ruin Theory and Credit Risk}

In this section we establish a relationship between ruin theory and credit risk by showing the relationship between the surplus process in ruin theory and solvency-ratio process in a structural credit risk model.

Dufresne and Gerber [18] extended the classical surplus process model in ruin theory by introducing a diffusion process $W(t)$ into the surplus process

$$
U(t)=u+c t+W(t)-S(t), \quad t \geq 0,
$$

where $U(t)$ is the time-t surplus, $u$ is the initial surplus, $c t$ is the premium income, and $S(t)$ is the aggregate loss. The aggregate loss $(S(t))_{t \geq 0}$ as $S(t)=X_{1}+X_{2}+\cdots+$ $X_{N_{t}}$ follows a compound Poisson process. Here, the random variables $X_{1}, X_{2}, \ldots, X_{N_{t}}$ are independent and identically distributed. The counting number process $\left(N_{t}\right)_{t \geq 0}$ follows a Poisson process with a parameter $\lambda$. The premium rate $c$ is 
set to be equal to $\lambda E[X](1+\theta)$, where $\theta>0$ represents the relative security loading. Ruin is triggered when the surplus falls below zero. $[W(t)]_{t \geq 0}$ is a Wiener process with drift 0 .

In credit risk literature, the trigger of default is defined when the firm value falls below its debt $D$, where $V_{0} \geq D$. In credit risk literature, the value of $\ln \left(V_{t} / D\right)$ is called the solvency ratio at time-t. If we let

$$
\begin{gathered}
u=\ln \left(\frac{V_{0}}{D}\right), \\
c=r-\frac{\sigma^{2}}{2}-\lambda_{\mu_{J}} \\
S(t)=\sum_{i=1}^{N_{t}} X_{i}=-\sum_{i=1}^{N_{t}} \ln Y_{i} \\
=-\sum_{i=1}^{N_{t}} \ln \left(J_{i}+1\right)=-\ln Y(t),
\end{gathered}
$$

then a solvency-ratio process is equivalent to the surplus process

$$
\begin{aligned}
\ln \left(\frac{V_{t}}{D}\right)= & \ln \left(\frac{V_{0}}{D}\right)+\left(r-\frac{\sigma^{2}}{2}-\lambda_{\mu_{J}}\right) t \\
& +\sigma Z_{t}+\sum_{i=1}^{N_{t}} \ln Y_{i} \\
= & u+c t+W(t)-S(t) \\
= & U(t),
\end{aligned}
$$

which is the relationship between ruin theory and credit risk.

The time of default or ruin is denoted by

$$
\tau=\inf \left\{t \mid U_{t}<0\right\}=\inf \left\{t \mid V_{t}<D\right\} .
$$

Conditional on the fact that a firm does not default at the current time- $t$, short-term default probability and survival probability can be given by

$$
\begin{aligned}
\varphi\left(\ln \left(\frac{V_{t}}{D}\right), T\right) & =Q\left\{\inf _{t<s \leq T} \ln \left(\frac{V_{s}}{D}\right)<0 \mid V_{t}>D\right\} \\
& =Q\left\{\inf _{t<s \leq T} V_{s}<D \mid V_{t}>D\right\} \\
& =Q\{t<\tau \leq T \mid \tau>t\}, \\
\varphi\left(\ln \left(\frac{V_{t}}{D}\right), T\right) & =Q\left\{\inf _{t<s \leq T} \ln \left(\frac{V_{s}}{D}\right) \geq 0 \mid V_{t}>D\right\} \\
& =Q\left\{\inf _{t<s \leq T} V_{s} \geq D \mid V_{t}>D\right\} \\
& =Q\{\tau \geq T \mid \tau>t\} .
\end{aligned}
$$

Since both of the surplus and the solvency-ratio process follow a jump-diffusion process, default can be triggered either by diffusion process or a jump event. When a default is caused by diffusion, we let $\tau_{d}, \varphi_{d}\left(\ln \left(V_{t} / D\right), T\right)$ and $\omega\left(\tau_{d}\right)=1$ represent the default time, the default probability, and the recovery rate separately. When a default is caused by a jump, the analogous parts are $\tau_{j}, \varphi_{j}\left(\ln \left(V_{t} / D\right), T\right)$ and $\omega\left(\tau_{j}\right) \leq 1$. By the total probability law, we have

$$
\varphi\left(\ln \left(\frac{V_{t}}{D}\right), T\right)=\varphi_{d}\left(\ln \left(\frac{V_{t}}{D}\right), T\right)+\varphi_{j}\left(\ln \left(\frac{V_{t}}{D}\right), T\right) .
$$

\section{The Credit Spread}

When a default occurs and is triggered by a jump event, which may cause a sudden drop in the firm value, the firm may suffer a deficit. The deficit $L\left(\tau_{j}\right)$ is defined by

$$
L\left(\tau_{j}\right)=-U\left(\tau_{j}\right)=-\ln \left(\frac{V_{\tau_{j}}}{D}\right) .
$$

When the firm is default because of a jump, the recovery rate can be rewritten as

$$
\omega\left(\tau_{j}\right)=\frac{V_{\tau_{j}}}{D}=\exp \left\{\ln \left(\frac{V_{\tau_{j}}}{D}\right)\right\}=\exp \left\{-L\left(\tau_{j}\right)\right\}
$$

Theorem 1. Under $R T V^{+}$scheme, if the firm value follows a jump-diffusion process and default occurs at any time before maturity $T$, then the yield spread is

$$
\begin{aligned}
R(t, T) & -r(t, T) \\
= & -\frac{1}{T-t} \ln \left\{1-\left[1-E_{t}^{\mathrm{Q}}\left[\omega^{2}\left(\tau_{j}\right)\right]\right] \varphi_{j}\left(\ln \left(\frac{V_{t}}{D}\right), T\right)\right\} \\
= & -\frac{1}{T-t} \ln \left\{1-\left[1-E_{t}^{\mathrm{Q}}\left[e^{2 L\left(\tau_{j}\right)}\right]\right] \varphi_{j}\left(\ln \left(\frac{V_{t}}{D}\right), T\right)\right\} .
\end{aligned}
$$

Proof. In general, we have

$$
\begin{aligned}
E_{t}^{Q}\left[\omega^{2}(\tau)\right] & \\
= & 1 \cdot Q\{\text { default caused by diffusion process }\} \\
& +E_{t}^{Q}\left[\omega^{2}\left(\tau_{j}\right)\right] \cdot Q\{\text { default caused by a jump }\} \\
= & \frac{\varphi_{d}\left(\ln \left(V_{t} / D\right), T\right)}{\varphi\left(\ln \left(V_{t} / D\right), T\right)}+E_{t}^{Q}\left[\omega^{2}\left(\tau_{j}\right)\right] \cdot \frac{\varphi_{j}\left(\ln \left(V_{t} / D\right), T\right)}{\varphi\left(\ln \left(V_{t} / D\right), T\right)} \\
= & \frac{\varphi_{d}\left(\ln \left(V_{t} / D\right), T\right)}{\varphi\left(\ln \left(V_{t} / D\right), T\right)}+E_{t}^{Q}\left[e^{-2 L\left(\tau_{j}\right)}\right] \cdot \frac{\varphi_{j}\left(\ln \left(V_{t} / D\right), T\right)}{\varphi\left(\ln \left(V_{t} / D\right), T\right)} .
\end{aligned}
$$


Substituting (23) into (10), we get

$$
\begin{aligned}
R(t, T) & -r(t, T) \\
= & -\frac{1}{T-t} \ln \left\{1-\left[1-E_{t}^{Q}\left[\omega^{2}(\tau)\right]\right] Q(\tau \leq T \mid \tau>t)\right\} \\
= & -\frac{1}{T-t} \ln \left\{1-\left[1-E_{t}^{Q}\left[\omega^{2}(\tau)\right]\right] \varphi\left(\ln \left(\frac{V_{t}}{D}\right), T\right)\right\} \\
= & -\frac{1}{T-t} \ln \left\{1-\varphi\left(\ln \left(\frac{V_{t}}{D}\right), T\right)+\varphi_{d}\left(\ln \left(\frac{V_{t}}{D}\right), T\right)\right. \\
& \left.\quad+E_{t}^{Q}\left[\omega^{2}\left(\tau_{j}\right)\right] \varphi_{j}\left(\ln \left(\frac{V_{t}}{D}\right), T\right)\right\} \\
= & -\frac{1}{T-t} \ln \left\{1-\varphi_{j}\left(\ln \left(\frac{V_{t}}{D}\right), T\right)\right. \\
= & \left.\quad+E_{t}^{Q}\left[\omega^{2}(\tau)\right] \varphi_{j}\left(\ln \left(\frac{V_{t}}{D}\right), T\right)\right\} \\
= & -\frac{1}{T-t} \ln \left\{1-\left[1-E_{t}^{Q}\left[\omega^{2}\left(\tau_{j}\right)\right]\right] \varphi_{j}\left(\ln \left(\frac{V_{t}}{D}\right), T\right)\right\} \\
= & {\left.\left[1-E_{t}^{Q}\left[e^{2 L\left(\tau_{j}\right)}\right]\right] \varphi_{j}\left(\ln \left(\frac{V_{t}}{D}\right), T\right)\right\} . }
\end{aligned}
$$

A joint distribution density function $f\left(x, l, t \mid V_{t}\right)$ of $U\left(\tau_{j}\right)$, the deficit at ruin $L\left(\tau_{j}\right)$, and the ruin time $\tau_{j}$ are used by Gerber and Shiu [19] and extended by Chen and Panjer [17]. The jump $X=-\ln Y$ is assumed to be independent and identically distributed with differentiable distribution function $P(x)$ and probability density function $p(x)$. Chen and Panjer [17] assumes that the jump size has a Logexponential probability density function $f(j)=\alpha(j+1)^{\alpha-1}$, $-1<j<0$.

According to Chen and Panjer [17], we have

$$
\begin{aligned}
E_{t}^{Q}\left[\omega^{2}\left(\tau_{j}\right)\right] \\
=E_{t}^{Q}\left[e^{-2 L\left(\tau_{j}\right)}\right] \\
=\frac{1}{\varphi_{j}\left(\ln \left(V_{t} / D\right), T\right)} \\
\quad \times \int_{t}^{T} \int_{0}^{\infty} \int_{0}^{\infty} e^{-2 l} f\left(x, l, t \mid V_{t}\right) d x d l d t \\
=\frac{1}{\varphi_{j}\left(\ln \left(V_{t} / D\right), T\right)} \\
\quad \times \int_{t}^{T} \int_{0}^{\infty} \int_{0}^{\infty} e^{-2 l} f\left(x, t \mid V_{t}\right) \frac{p(x+l)}{1-P(x)} d x d l d t .
\end{aligned}
$$

If the jump size follows a Log-exponential distribution, then the corresponding jump distribution $L=-\ln (J+$ 1) possesses an exponential distribution with probability density $p(l)=\beta e^{-\beta l}$. is

Thus, the probability density function of the recovery rate

$$
f_{\varpi(\tau)}(x)= \begin{cases}\frac{\varphi_{d}\left(\ln \left(V_{t} / D\right), T\right)}{\varphi\left(\ln \left(V_{t} / D\right), T\right)}, & x=1, \\ \frac{\varphi_{j}\left(\ln \left(V_{t} / D\right), T\right)}{\varphi\left(\ln \left(V_{t} / D\right), T\right)} f_{\varpi\left(\tau_{j}\right)}(x), & 0<x<1,\end{cases}
$$

where $f_{\varpi\left(\tau_{j}\right)}(x)=\beta x^{\beta-1}, 0<x<1$.

Theorem 2. Under $R T V^{+}$scheme, assume that the firm value follows a jump-diffusion process and default occurs at any time before maturity $T$. If the jump follows an exponential distribution as $p_{l}(x)=\beta e^{-\beta x}$, it holds that

$$
\begin{aligned}
& \text { (1) } E_{t}^{Q}\left[\omega^{2}\left(\tau_{j}\right)\right]=\frac{\beta}{\beta+2}, \\
& \text { (2) } E_{t}^{Q}\left[\omega^{2}(\tau)\right]=1-\frac{2}{\beta+2} \cdot \frac{\varphi_{j}\left(\ln \left(V_{t} / D\right), T\right)}{\varphi\left(\ln \left(V_{t} / D\right), T\right)},
\end{aligned}
$$

(3) $R(t, T)-r(t, T)$

$$
\begin{array}{r}
=-\frac{1}{T-t} \ln \left\{1-\frac{\beta}{\beta+2} \cdot \varphi_{j}\left(\ln \left(\frac{V_{t}}{D}\right), T\right)\right\}, \\
\text { (4) } \frac{P(t, T)-V(t, T)}{P(t, T)}=\frac{\beta}{\beta+2} \cdot \varphi_{j}\left(\ln \left(\frac{V_{t}}{D}\right), T\right) .
\end{array}
$$

Proof. If default is caused by a jump, one has

$$
\begin{aligned}
E_{t}^{Q}\left[\omega^{2}\left(\tau_{j}\right)\right] \\
=\frac{1}{\varphi_{j}\left(\ln \left(V_{t} / D\right), T\right)} \\
\quad \times \int_{t}^{T} \int_{0}^{\infty} \int_{0}^{\infty} e^{-2 l} f\left(x, t \mid V_{t}\right) \frac{p(x+l)}{1-P(x)} d x d l d t \\
=\frac{1}{\varphi_{j}\left(\ln \left(V_{t} / D\right), T\right)} \\
\quad \times \int_{t}^{T} \int_{0}^{\infty} f\left(x, t \mid V_{t}\right) d x d t \int_{0}^{\infty} e^{-2 l} \frac{\beta e^{-\beta(x+l)}}{e^{-\beta x}} d l \\
=\frac{1}{\varphi_{j}\left(\ln \left(V_{t} / D\right), T\right)} \varphi_{j}\left(\ln \left(\frac{V_{t}}{D}\right), T\right) \int_{0}^{\infty} \beta e^{-(\beta+2) l} d l \\
=\frac{\beta}{\beta+2} .
\end{aligned}
$$


Substituting (28) into (23) gives rise to

$$
\begin{aligned}
E_{t}^{Q}\left[\omega^{2}(\tau)\right] & \\
= & \frac{\varphi_{d}\left(\ln \left(V_{t} / D\right), T\right)}{\varphi\left(\ln \left(V_{t} / D\right), T\right)}+\frac{\beta}{\beta+2} \cdot \frac{\varphi_{j}\left(\ln \left(V_{t} / D\right), T\right)}{\varphi\left(\ln \left(V_{t} / D\right), T\right)} \\
= & \frac{\varphi_{d}\left(\ln \left(V_{t} / D\right), T\right)}{\varphi\left(\ln \left(V_{t} / D\right), T\right)}+\frac{\varphi_{j}\left(\ln \left(V_{t} / D\right), T\right)}{\varphi\left(\ln \left(V_{t} / D\right), T\right)} \\
& -\frac{2}{\beta+2} \cdot \frac{\varphi_{j}\left(\ln \left(V_{t} / D\right), T\right)}{\varphi\left(\ln \left(V_{t} / D\right), T\right)} \\
= & 1-\frac{2}{\beta+2} \cdot \frac{\varphi_{j}\left(\ln \left(V_{t} / D\right), T\right)}{\varphi\left(\ln \left(V_{t} / D\right), T\right)} .
\end{aligned}
$$

From (24), the credit spread is written as

$$
\begin{aligned}
& R(t, T)-r(t, T) \\
&=-\frac{1}{T-t} \ln \left\{1-\left(1-E_{t}^{Q}\left[\omega^{2}(\tau)\right]\right) \varphi\left(\ln \left(\frac{V_{t}}{D}\right), T\right)\right\} \\
&=-\frac{1}{T-t} \ln \left\{1-\frac{\beta}{\beta+2} \cdot \frac{\varphi_{j}\left(\ln \left(V_{t} / D\right), T\right)}{\varphi\left(\ln \left(V_{t} / D\right), T\right)}\right. \\
&\left.\cdot \varphi\left(\ln \left(\frac{V_{t}}{D}\right), T\right)\right\} \\
&=-\frac{1}{T-t} \ln \left\{1-\frac{\beta}{\beta+2} \cdot \varphi_{j}\left(\ln \left(\frac{V_{t}}{D}\right), T\right)\right\} .
\end{aligned}
$$

From (9) we have

$$
\begin{aligned}
& \frac{P(t, T)}{P(}-V(t, T) \\
&= 1-\frac{V(t, T)}{P(t, T)} \\
&=\left\{1-E_{t}^{Q}\left[\omega^{2}(\tau)\right]\right\} \varphi\left(\ln \left(\frac{V_{t}}{D}\right), T\right) \\
&=\left\{1-\frac{\varphi_{d}\left(\ln \left(V_{t} / D\right), T\right)}{\varphi\left(\ln \left(V_{t} / D\right), T\right)}\right. \\
& \times \varphi\left(\ln \left(\frac{V_{t}}{D}\right), T\right) \\
&= \varphi\left(\ln \left(\frac{V_{t}}{D}\right), T\right)-\varphi_{d}\left(\ln \left(\frac{V_{t}}{D}\right), T\right)-E_{t}^{Q}\left[e^{-2 L\left(\tau_{j}\right)}\right] \\
& \cdot \varphi_{j}\left(\ln \left(\frac{V_{t}}{D}\right), T\right) \\
&= \varphi_{j}\left(\ln \left(\frac{V_{t}}{D}\right), T\right)-E_{t}^{Q}\left[e^{-2 L\left(\tau_{j}\right)}\right] \cdot \varphi_{j}\left(\ln \left(\frac{\left.\left.V_{t} / D\right), T\right)}{D}\right), T\right)
\end{aligned}
$$

$$
\begin{aligned}
& =\left\{1-E_{t}^{Q}\left[e^{-2 L\left(\tau_{j}\right)}\right]\right\} \cdot \varphi_{j}\left(\ln \left(\frac{V_{t}}{D}\right), T\right) \\
& =\frac{\beta}{\beta+2} \cdot \varphi_{j}\left(\ln \left(\frac{V_{t}}{D}\right), T\right) .
\end{aligned}
$$

The proof of Theorem 2 is completed.

\section{Multiple Seniorities Cases}

In corporate debt market, a company may issue debts with multiple seniorities. Under multiple seniorities cases, the strict priority rule is applied, which means that once a default occurs, the junior claimants can be paid until the senior claimants are fully paid off. Suppose that $n$ seniority classes of bonds are issued by a firm, and the proportion of each class is denoted by $p_{1}, p_{2}, \ldots, p_{n}$, with $\sum_{k=1}^{n} p_{k}=1$. The recovery rate for each class, $\Phi_{1}(\tau), \omega_{2}(\tau), \ldots, \omega_{n}(\tau)$, is given by

$$
\begin{gathered}
\omega_{1}(\tau)=\min \left(\frac{\omega(\tau)}{p_{1}}, 1\right), \\
\omega_{i}(\tau)=\min \left(\frac{\left(\omega(\tau)-\sum_{k=1}^{i-1} p_{k}\right)_{+}}{p_{i}}, 1\right), \\
i=1,2, \ldots,(n-1), \\
\omega_{n}(\tau)=\frac{\left(\omega(\tau)-\sum_{k=1}^{n-1} p_{k}\right)_{+}}{p_{n}},
\end{gathered}
$$

where ()$_{+}$denotes the positive value of () .

Theorem 3. If the firm-value process follows a jump-diffusion process and default occurs at any time before maturity $T$, and if $\mathrm{RTV}^{+}$scheme and the strict priority rule are applied, then the following equations are valid under the assumption that the jump follows an exponential distribution $p(l)=\beta e^{-\beta l}$ :

(1) $E_{t}^{Q}\left[\omega_{1}^{2}\left(\tau_{j}\right)\right]=1-\frac{2}{\beta+2} \cdot p_{1}^{\beta}$,
(2) $E_{t}^{Q}\left[\omega_{i}^{2}\left(\tau_{j}\right)\right]=1-\frac{1}{p_{i}^{2}} \frac{3}{(\beta+2)(\beta+1)}$

$$
\times\left[\left(\sum_{k=1}^{i} p_{k}\right)^{\beta+2}-\left(\sum_{k=1}^{i-1} p_{k}\right)^{\beta+2}\right]
$$$$
j=1,2, \ldots, n-1,
$$

(3) $E_{t}^{Q}\left[\omega_{n}^{2}\left(\tau_{j}\right)\right]=\frac{1}{p_{n}^{2}}\left\{1-2 p_{n}+p_{n}^{2}-\frac{\beta(\beta+3)}{(\beta+2)(\beta+1)}\right.$

$$
\left.-\frac{2}{(\beta+2)(\beta+1)}\left(\sum_{k=1}^{n-1} p_{k}\right)^{\beta+2}\right\} \text {. }
$$


Proof. For the highest seniority class, we have

$$
\begin{aligned}
E_{t}^{Q}\left[\Phi_{1}^{2}\left(\tau_{j}\right)\right] & =E_{t}^{Q}\left[\left\{\min \left(\frac{\Phi^{2}\left(\tau_{j}\right)}{p_{1}}, 1\right)\right\}^{2}\right] \\
& =\frac{1}{p_{1}^{2}} \int_{0}^{p_{1}} x^{2} \cdot \beta x^{\beta-1} d x+\int_{p_{1}}^{1} 1 \cdot \beta x^{\beta-1} d x \\
& =\frac{1}{p_{1}^{2}} \cdot \frac{\beta}{\beta+2} \cdot\left[x^{\beta+2}\right]_{0}^{p_{1}}+\left[x^{\beta}\right]_{p_{1}}^{1} \\
& =1-\frac{2}{\beta+2} \cdot p_{1}^{\beta} .
\end{aligned}
$$

For the lower seniority classes, we get

$$
\begin{aligned}
& E_{t}^{\mathrm{Q}}\left[\varpi_{i}^{2}\left(\tau_{j}\right)\right] \\
& =E_{t}^{Q}\left[\min \left(\frac{\left(\varrho(\tau)-\sum_{k=1}^{i-1} p_{k}\right)_{+}}{p_{i}}, 1\right)\right]^{2} \\
& =\frac{1}{p_{i}^{2}} \int_{0}^{\sum_{k=1}^{i-1} p_{k}} 0 \cdot \beta x^{\beta-1} d x \\
& +\frac{1}{p_{i}^{2}} \int_{\sum_{k=1}^{i-1} p_{k}}^{\sum_{k=1}^{i} p_{k}}\left(x-\sum_{k=1}^{i-1} p_{k}\right)^{2} \cdot \beta x^{\beta-1} d x \\
& +\int_{\sum_{k=1}^{i} p_{k}}^{1} 1 \cdot \beta x^{\beta-1} d x \\
& =\frac{1}{p_{i}^{2}} \cdot \int_{\sum_{k=1}^{i-1} p_{k}}^{\sum_{k=1}^{i} p_{k}} \beta x^{\beta+1} d x \\
& -2 \cdot \frac{1}{p_{i}^{2}} \cdot \sum_{k=1}^{i-1} p_{k} \cdot \int_{\sum_{k=1}^{i-1} p_{k}}^{\sum_{k=1}^{i} p_{k}} \beta x^{\beta} d x \\
& +\frac{1}{p_{i}^{2}} \cdot\left(\sum_{k=1}^{i-1} p_{k}\right)^{2} \cdot \int_{\sum_{k=1}^{i-1} p_{k}}^{\sum_{k=1}^{i} p_{k}} \beta x^{\beta-1} d x \\
& +\int_{\sum_{k=1}^{i} p_{k}}^{1} 1 \cdot \beta x^{\beta-1} d x \\
& =\frac{1}{p_{i}^{2}} \cdot \frac{\beta}{\beta+2} \cdot\left[\left(\sum_{k=1}^{i} p_{k}\right)^{\beta+2}-\left(\sum_{k=1}^{i-1} p_{k}\right)^{\beta+2}\right] \\
& -2 \cdot \frac{1}{p_{i}^{2}} \cdot \sum_{k=1}^{i-1} p_{k} \cdot \frac{\beta}{\beta+1} \\
& {\left[\left(\sum_{k=1}^{i} p_{k}\right)^{1+\beta}-\left(\sum_{k=1}^{i-1} p_{k}\right)^{1+\beta}\right]} \\
& +\frac{1}{p_{i}^{2}} \cdot\left(\sum_{k=1}^{i-1} p_{k}\right)^{2}\left[\left(\sum_{k=1}^{i} p_{k}\right)^{\beta}-\left(\sum_{k=1}^{i-1} p_{k}\right)^{\beta}\right]
\end{aligned}
$$

$$
\begin{aligned}
& +\left[1-\left(\sum_{k=1}^{i-1} p_{k}\right)^{\beta}\right] \\
= & 1-\frac{1}{p_{i}^{2}} \frac{3}{(\beta+2)(\beta+1)} \\
& \times\left[\left(\sum_{k=1}^{i} p_{k}\right)^{\beta+2}-\left(\sum_{k=1}^{i-1} p_{k}\right)^{\beta+2}\right], \\
j & =2,3, \ldots, n-1 .
\end{aligned}
$$

For the lowest seniority class, it yields

$$
\begin{aligned}
& \omega_{n}^{2}\left(\tau_{j}\right)=E_{t}^{Q}\left[\left(\frac{\left(\omega\left(\tau_{j}\right)-\sum_{k=1}^{n-1} p_{k}\right)_{+}}{p_{n}}\right)^{2}\right] \\
& =\frac{1}{p_{n}^{2}}\left\{\int_{0}^{\sum_{k=1}^{n-1} p_{k}} 0 \cdot \beta x^{\beta-1} d x\right. \\
& \left.+\int_{\sum_{k=1}^{n-1} p_{k}}^{1}\left(x-\sum_{k=1}^{n-1} p_{k}\right)^{2} \cdot \beta x^{\beta-1} d x\right\} \\
& =\frac{1}{p_{n}^{2}}\left\{\int_{\sum_{k=1}^{n-1} p_{k}}^{1} \beta x^{\beta+1}-2 \beta \cdot\left(\sum_{k=1}^{n-1} p_{k}\right)\right. \\
& \left.\cdot x^{\beta}+\beta \cdot\left(\sum_{k=1}^{n-1} p_{k}\right)^{2} \cdot x^{\beta-1} d x\right\} \\
& =\frac{1}{p_{n}^{2}} \cdot \frac{\beta}{\beta+2} \cdot\left(x^{\beta+2}\right)_{\sum_{k=1}^{n-1} p_{k}}^{1} \\
& -\frac{1}{p_{n}^{2}} \cdot \frac{2 \beta}{\beta+1} \cdot\left(\sum_{k=1}^{n-1} p_{k}\right) \cdot\left(x^{\beta+1}\right)_{\sum_{k=1}^{n-1} p_{k}}^{1} \\
& +\frac{1}{p_{n}^{2}} \cdot\left(\sum_{k=1}^{n-1} p_{k}\right)^{2} \cdot\left(x^{\beta}\right)_{\sum_{k=1}^{n-1} p_{k}}^{1} \\
& =\frac{1}{p_{n}^{2}} \cdot \frac{\beta}{\beta+2} \cdot\left[1-\left(\sum_{k=1}^{n-1} p_{k}\right)^{\beta+2}\right]-\frac{1}{p_{n}^{2}} \\
& \cdot \frac{2 \beta}{\beta+1} \cdot\left(\sum_{k=1}^{n-1} p_{k}\right) \cdot\left[1-\left(\sum_{k=1}^{n-1} p_{k}\right)^{\beta+1}\right] \\
& +\frac{1}{p_{n}^{2}} \cdot\left(\sum_{k=1}^{n-1} p_{k}\right)^{2} \cdot\left[1-\left(\sum_{k=1}^{n-1} p_{k}\right)^{\beta}\right] \\
& =\frac{1}{p_{n}^{2}}\left\{1-2 p_{n}+p_{n}^{2}-\frac{\beta(\beta+3)}{(\beta+1)(\beta+2)}\right. \\
& \left.-\frac{2}{(\beta+1)(\beta+2)}\left(\sum_{k=1}^{n-1} p_{k}\right)^{\beta+2}\right\} \text {. }
\end{aligned}
$$

The proof of Theorem 3 is completed. 


\section{The Implied Jump Size Distribution and the Optimal Default Probability}

In this section, we will find the implied jump distribution and the optimal default probability by assuming that the credit spread of the jump-diffusion model is in accord with the market credit spread.

For simplicity of presentation, Chen and Panjer [17] assume that a company issues bonds of only two seniority classes with the same maturity time $T$. The proportions of the higher and lower seniority classes of bonds are denoted by $p_{1}$ and $1-p_{1}$, respectively. The recovery rates for the higher and lower seniority are $\omega_{1}(\tau)=\min \left(\omega(\tau) / p_{1}, 1\right)$ and $\omega_{2}(\tau)=\left(\omega(\tau)-p_{1}\right)_{+} /\left(1-p_{1}\right)$.

From Theorem 3, we have

$$
\begin{aligned}
& E_{t}^{Q}\left[\omega_{1}^{2}\left(\tau_{j}\right)\right]=E_{t}^{Q}\left[\left(\min \left(\frac{\omega^{2}\left(\tau_{j}\right)}{p_{1}}, 1\right)\right)^{2}\right] \\
& =\frac{1}{p_{1}^{2}} \int_{0}^{p_{1}} x^{2} \cdot \beta x^{\beta-1} d x+\int_{p_{1}}^{1} 1 \cdot \beta x^{\beta-1} d x \\
& =1-\frac{2}{\beta+2} \cdot p_{1}^{\beta} \text {, } \\
& E_{t}^{Q}\left[\omega_{2}^{2}\left(\tau_{j}\right)\right]=E_{t}^{Q}\left[\left(\frac{\left(\omega\left(\tau_{j}\right)-p_{1}\right)_{+}}{1-p_{1}}\right)^{2}\right] \\
& =\frac{1}{\left(1-p_{1}\right)^{2}} \cdot \int_{0}^{p_{1}} 0 \beta x^{\beta-1} d x \\
& +\frac{1}{\left(1-p_{1}\right)^{2}} \int_{p_{1}}^{1}\left(x-p_{1}\right)^{2} \cdot \beta x^{\beta-1} d x \\
& =\frac{1}{\left(1-p_{1}\right)^{2}} \cdot \int_{p_{1}}^{1}\left(\beta x^{\beta+1}-2 p_{1} \beta x^{\beta}\right. \\
& \left.+p_{1}^{2} \beta x^{\beta-1}\right) d x \\
& =\frac{1}{\left(1-p_{1}\right)^{2}} \cdot\left[\frac{\beta}{\beta+2} \cdot\left(1-p_{1}^{\beta+2}\right)-\frac{2 p_{1} \beta}{\beta+1}\right. \\
& \left.\cdot\left(1-p_{1}^{\beta+1}\right)+p_{1}^{2} \cdot\left(1-p_{1}^{\beta}\right)\right] \\
& =\frac{1}{\left(1-p_{1}\right)^{2}} \cdot\left[\left(\frac{\beta}{\beta+2}-\frac{2 \beta}{\beta+1} p_{1}+p_{1}^{2}\right)\right. \\
& \left.-\frac{2}{(\beta+1)(\beta+2)} p_{1}^{\beta+2}\right] \text {. }
\end{aligned}
$$

From (31), for both of the two seniority classes of bonds, we have

$$
\frac{P(t, T)-V_{1}(t, T)}{P(t, T)}=\left\{1-E_{t}^{Q}\left[\omega_{1}^{2}\left(\tau_{j}\right)\right]\right\} \cdot \varphi_{j}\left(\ln \left(\frac{V_{t}}{D}\right), T\right),
$$

$$
\frac{P(t, T)-V_{2}(t, T)}{P(t, T)}=\left\{1-E_{t}^{Q}\left[\omega_{2}^{2}\left(\tau_{j}\right)\right]\right\} \cdot \varphi_{j}\left(\ln \left(\frac{V_{t}}{D}\right), T\right) .
$$

It follows from (38) and (39) that

$$
\frac{P(t, T)-V_{1}(t, T)}{P(t, T)-V_{2}(t, T)}=\frac{1-E_{t}^{\mathrm{Q}}\left[\omega_{1}^{2}\left(\tau_{j}\right)\right]}{1-E_{t}^{\mathrm{Q}}\left[\omega_{2}^{2}\left(\tau_{j}\right)\right]}
$$

Note that if we consider the three equations (37) and (40), we have three unknown variables. It is clear that the parameter $\beta$ can be solved. Thus, from (38), the default probability caused by a jump is expressed by

$$
\begin{aligned}
\varphi_{j}\left(\ln \left(\frac{V_{t}}{D}\right), T\right) \\
=\left\{1-E_{t}^{\mathrm{Q}}\left[\omega_{1}^{2}\left(\tau_{j}\right)\right]\right\}^{-1} \cdot \frac{P(t, T)-V_{1}(t, T)}{P(t, T)} \\
=\frac{\beta+2}{2 p_{1}^{\beta}} \cdot \frac{P(t, T)-V_{1}(t, T)}{P(t, T)} .
\end{aligned}
$$

We have considered the situation that a firm issues bonds of only two seniorities with the same maturity time and obtained the implied jump distribution and the default probability caused by a jump. When a firm issues bonds of more than two seniorities, we may have more than three equations. In this case, we can improve Assumption II in Section 2 where $(T-\widehat{\tau})$ is a function with parameters of the recovery rate $\omega(\tau)$. For instance, if a firm issues bonds of three seniorities classes, we can assume that $T-\widehat{\tau}=-(1 / r) \ln (a$. $\omega(\tau))$. When a firm issues bonds of $m(m \geq 2)$ seniorities classes, we assume the function to be a $(m-1)$-parameter edition.

\section{Conclusion}

In this paper, we have studied and tried to lay a theoretical groundwork for a credit risk valuation model with timecontinuous liquidation based on ruin theory. We develop a recovery scheme, named $\mathrm{RTV}^{+}$scheme, which is an extension of the recovery of treasury value scheme (RTV) with time-continuous liquidation. By using the function of the optimal time for liquidation and the recovery rate, we obtain the function expression of the risky bond price. In addition, a relationship between credit risk and ruin theory is constructed by using equivalence principle. Thus, the methodology in ruin theory can be used in credit risk valuation. Specifically, when the firm value follows a jumpdiffusion process with a Log-exponentially distributed jump, we find a method to obtain the optimal default probability with time-continuous liquidation.

\section{Conflict of Interests}

The authors declare that there is no conflict of interests regarding the publication of this paper.

\section{Acknowledgment}

This work is supported by the Fundamental Research Funds for the Central Universities (JBK1207004, JBK120504, and JBK130401). 


\section{References}

[1] R. C. Merton, "On the pricing of corporate debt: the risk structure of interest rates," The Journal of Finance, vol. 29, no. 2, pp. 449-470, 1974.

[2] R. C. Merton, "Option pricing when underlying stock returns are discontinuous," Journal of Financial Economics, vol. 3, no. 1-2, pp. 125-144, 1976.

[3] F. Black and J. C. Cox, "Valuing corporate securities: some effects of bond indenture provision," The Journal of Finance, vol. 31, no. 2, pp. 351-367, 1976.

[4] F. A. Lonigstaff and E. S. Schwartz, "A simple approach to valuing risky fixed and floating rate debt," The Journal of Finance, vol. 50, no. 3, pp. 789-819, 1995.

[5] H. E. Leland, "Presendential address: agency costs, risk management, and capital structure," Journal of Finance, vol. 53, no. 4, pp. 1213-1243, 1998.

[6] E. P. Jones, S. P. Mason, and E. Rosenfeld, "Contingent claims analysis of corporate capital structure: an empirical investigation," The Journal of Finance, vol. 39, pp. 611-627, 1984.

[7] O. Sarig and A. Warga, "Some empirical estimates of the risk structure of interest rates," Journal of Finance, vol. 44, no. 5, pp. 1351-1360, 1989.

[8] J. S. Fons, "Using default rates to model the term structure of credit risk," Financial Analysts Journal, vol. 50, no. 5, pp. 25-32, 1994.

[9] C. Zhou, "The term structure of credit spreads with jump risk," Journal of Banking and Finance, vol. 25, no. 11, pp. 2015-2040, 2001.

[10] P. Artzner and F. Delbaen, "Default risk insurance and incomplete markets," Mathematical Finance, vol. 5, no. 3, pp. 187-195, 1995.

[11] R. A. Jarrow and S. M. Turnbull, "Pricing derivatives on financial securities subject to credit risk," The Journal of Finance, vol. 50, no. 1, pp. 53-86, 1995.

[12] R. A. Jarrow, D. Lando, and S. M. Turnbull, "A markov model for the term structure of credit risk spreads," The Review of Financial Studies, vol. 10, no. 2, pp. 481-523, 1997.

[13] D. Li, "Constructing a credit curve," in Credit Risk: Risk Special Report, pp. 40-44, 1998.

[14] D. B. Madan and H. Unal, "Pricing the risks of default," Review of Derivatives Research, vol. 2, no. 2-3, pp. 121-160, 1998.

[15] C.-J. Chen and H. Panjer, "Unifying discrete structural models and reduced-form models in credit risk using a jump-diffusion process," Insurance, vol. 33, no. 2, pp. 357-380, 2003.

[16] H. Yang, "Ruin theory in a financial corporation model with credit risk," Insurance, vol. 33, no. 1, pp. 135-145, 2003.

[17] C.-J. Chen and H. Panjer, "A bridge from ruin theory to credit risk," Review of Quantitative Finance and Accounting, vol. 32, no. 4, pp. 373-403, 2009.

[18] F. Dufresne and H. U. Gerber, "Risk theory for the compound Poisson process that is perturbed by diffusion," Insurance, vol. 10, no. 1, pp. 51-59, 1991.

[19] H. U. Gerber and E. S. W. Shiu, "The joint distribution of the time of ruin, the surplus immediately before ruin, and the deficit at ruin," Insurance, vol. 21, no. 2, pp. 129-137, 1997. 


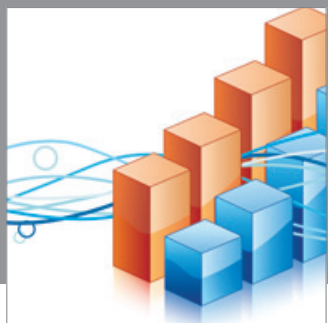

Advances in

Operations Research

mansans

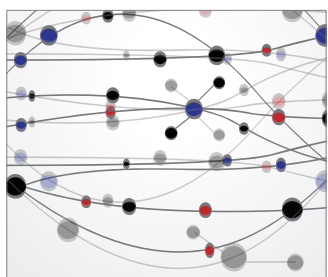

The Scientific World Journal
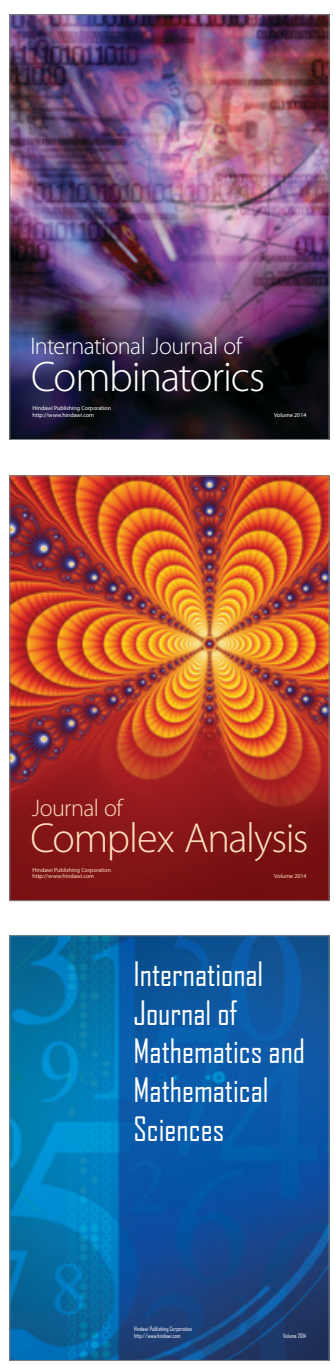
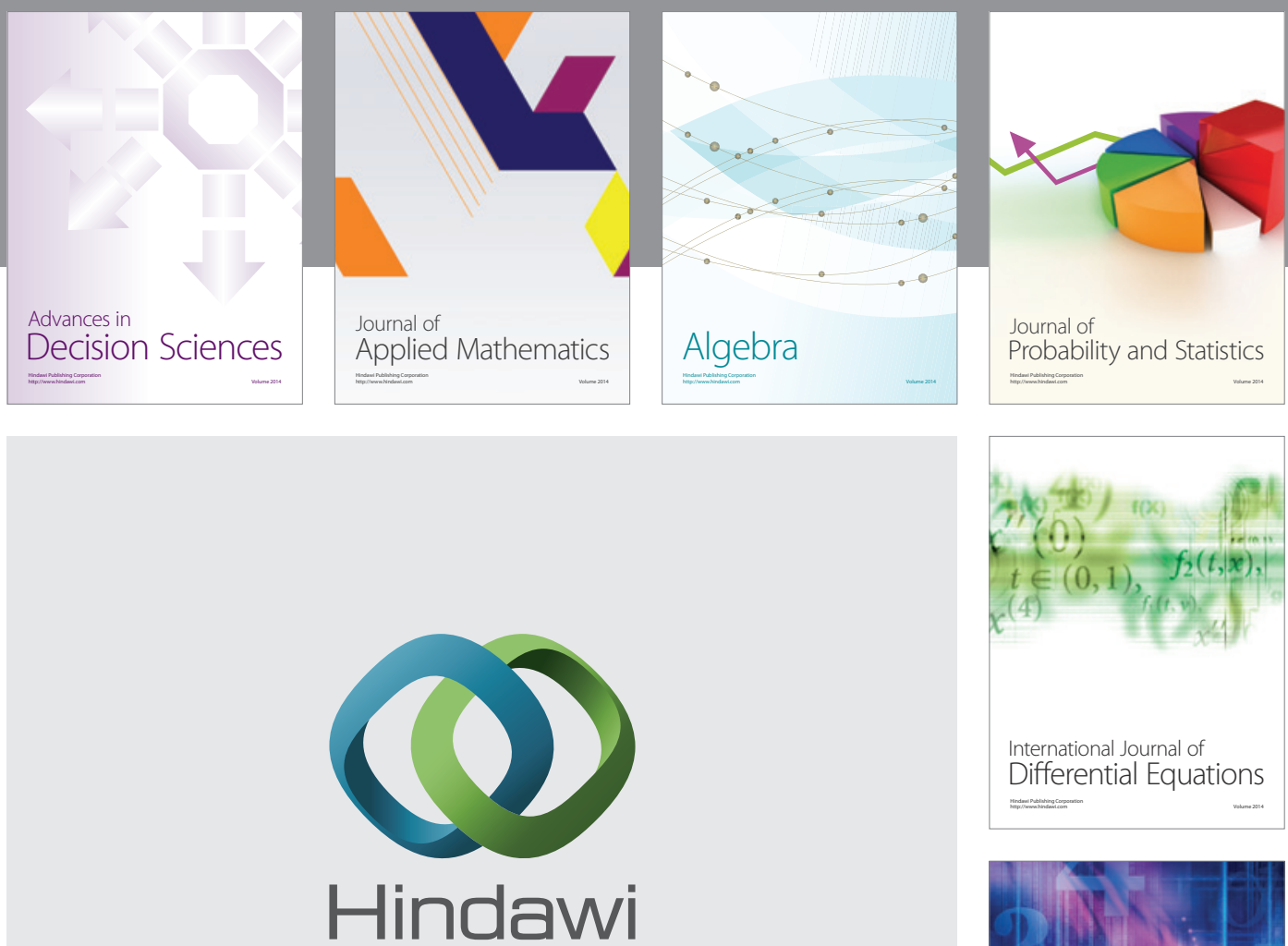

Submit your manuscripts at http://www.hindawi.com
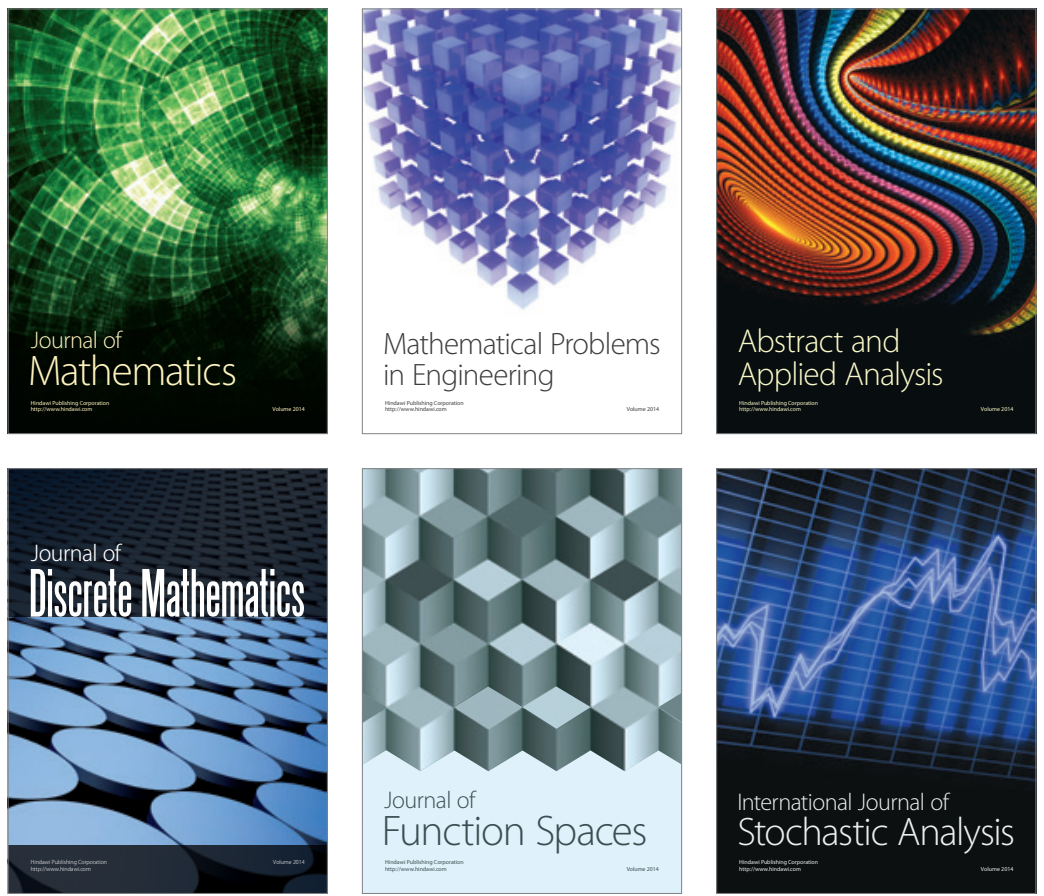

Journal of

Function Spaces

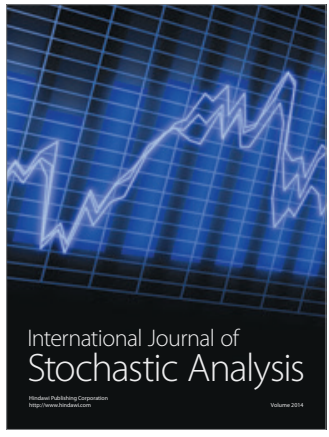

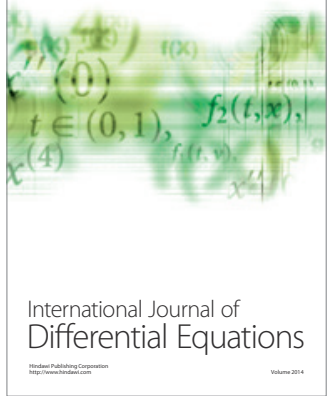
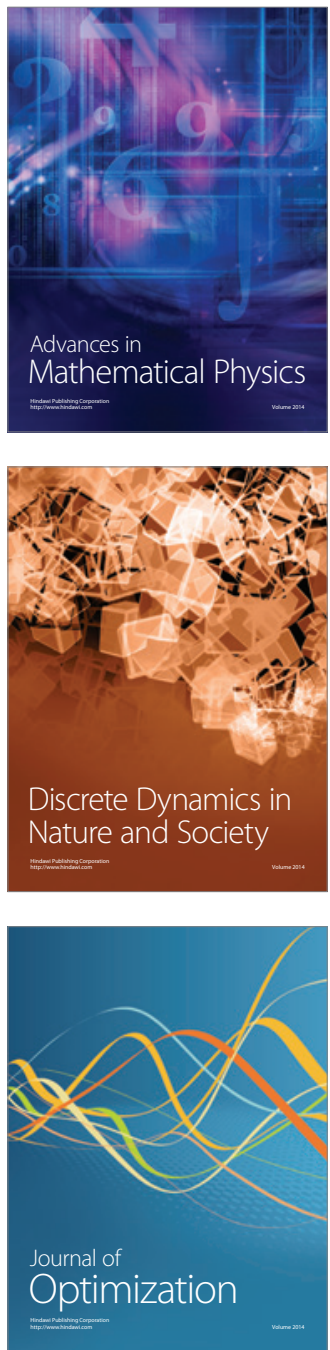